\title{
Intravitreal Injection of Ranibizumab in Macular Edema Secondary to Retinal Vein Occlusion
}

\author{
Sara A Elbadi, Walid M Gaafar, Mohamed S Elwan, Asaad A Ghanem* \\ Mansoura Ophthalmology Center, Faculty of Medicine, Mansoura University, Egypt \\ *Corresponding author: Asaad A. Ghanem, Mansoura Ophthalmic Center , Faculty of Medicine, Mansoura University, Mansoura, Egypt
}

\begin{abstract}
Aim: This study aimed to evaluate the safety and efficacy of intravitreal Ranibizumab $0.5 \mathrm{mg}$ in the treatment of macular edema secondary to retinal vein occlusion.

Patients \& Methods: This was a prospective interventional analytical study included 39 eyes of 39 patients with retinal vein occlusion. Ophthalmic examination included assessment of visual acuity, measurement of intraocular pressure, and fundus examination. All patients were scanned using Swept source optical coherence tomography (3D DRI OCT Triton [plus], Topcon Corporation, Tokyo, Japan) to assess central macular thickness. The changes of visual acuity, IOP, and central macular thickness were assessed. Data were analyzed via Kolmogorov-Smirnov test and Wilcoxon signed rank.

Results: The mean age was $56.56 \pm 9.6,48.7 \%$ were male and $51.3 \%$ were females. Hypertension was detected in $69.2 \%$, and hyperlipidemia in $2.6 \%$. The mean best corrected visual acuity was $1.5 \log$ MAR, $1.00 \operatorname{logMAR}, 1.00$ logMAR, preoperative, fourth month, six months postoperative, respectively, $(\mathrm{p}<0.001)$. The mean central macular thickness was $675 \mu, 306 \mathrm{u}, 264 \mathrm{u}$, preoperative, fourth month, six months postoperative, respectively, ( $\mathrm{p}<0.001)$. The OP was $16.5 \mathrm{mmHg}, 16.9 \mathrm{mmHg}, 17.1 \mathrm{mmHg}$, preoperative, fourth month, six months postoperative, respectively, $(\mathrm{p}=0.423)$. There were no observed significant ocular adverse events such as ocular inflammation, sterile and infectious endophthalmitis, or sustained increase in intraocular pressure with the use of intravitreal ranizumab injections.
\end{abstract}

Conclusion: Intravitreal Ranibizumab injections as monotherapy have shown promising results with BCVA improvement and a decrease of central macular thickness in patients with macular edema secondary to retinal vein occlusion.

Keywords: Ranibizumab; Macular Thickness; OCT; Visual Acuity

\section{Introduction}

Retinal vein occlusion (RVO) is the most common retinal vascular disease after diabetic retinopathy [1]. Depending on the area of retinal venous drainage effectively occluded it is broadly classified as either central retinal vein occlusion (CRVO), hemispheric retinal vein occlusion, or branch retinal vein occlusion (BRVO) [2]. Although the exact etiology of RVO remains elusive, it is likely to follow a thrombotic event. In CRVO this may occur in the central retinal vein (CRV) at the lamina cribrosa or at a variable distance in its journey within the optic nerve posterior to the lamina cribrosa [2]. Hypoxia-induced expression of vascular endothelial growth factor (VEGF) is thought to be a trigger for macular edema. High intravitreal levels of VEGF have been found in patients with retinal vein occlusion [3]. Upregulation of VEGF is associated with breakdown of the blood-retina barrier with increased vascular permeability resulting in retinal edema, stimulation of endothelial cell growth, and neovascularization [4,5]. Macular edema leads to vision loss in many patients with either central or branch retinal vein occlusions (CRVO or BRVO). BRVO is the more common of the two presentations, accounting for approximately $80 \%$ of RVO [6].

Recently, there has been interest in the use of vascular endothelial growth factor (VEGF) inhibition in the treatment of RVO because of the observation of increased VEGF in the vitreous and aqueous of patients with these conditions [7]. 


\section{Patients and Methods}

\section{Study Population}

This was a prospective interventional analytical study conducted at Mansoura ophthalmic center, Mansoura university. The study protocol approved by medical research ethics committee, faculty of medicine, Mansoura University (code number: MS/16.02.108). Informed consent was obtained from each participant in the study after assuring confidentiality.

\section{Inclusion criteria}

Included patients older than 18 , both gender, patients with clinically significant macular edema secondary to retinal vein occlusion and central macular thickness (CMT) was $>250$ um by optical coherence tomography.

\section{Exclusion Criteria}

Included patients with macular scar, macular hole, uveitis, neovascular glaucoma, age related macular degeneration, diabetic macular edema, patients had undergone treatment for macular edema secondary to retinal vein occlusion triamcinolone and vitrectomy. Also, patient has relevant malignant systemic disease, and media opacity that does not permit optical coherence tomography acquisition with good signal strength were excluded.

\section{Ocular Examination}

All subjects underwent an ophthalmic examination including assessment of visual acuity using Snellen chart at 6 meter distance and converted to log MAR, anterior segment evaluation using slit lamp biomicroscope (Haag Streit BP 900) (Haag-Streit, Koeniz, Switzerland), refraction using auto-refractometer (Topcon , KR800), intraocular pressure (IOP) measurement using Goldman applanation tonometry, Fundus examination using slit lamp bio microscopy using non-contact Volk lens +78 D or +90 D, Ocular coherence tomography imaging for CMT (central macular thickness).

\section{Swept Source OCT Imaging}

Three dimensional deep range imaging OCT Triton Plus (3D DRI OCT TRITON [plus],Topcon Corporation, Tokyo, Japan) with a high speed of 100,000 axial scans/s and center wavelength of 1,050 $\mathrm{nm}$ (version 10.07),digital and optical axial resolution of $2.6 \mu \mathrm{m}$ and $8 \mu \mathrm{m}$ in tissue, respectively and transverse resolution of $20 \mu \mathrm{m}$. The steps of scanning were done as follows, Mydriatic ( tropicamide $1 \%$ ) eye drop used to achieve a pupil dilatation to assure maximal OCT signal and analysis in patients prior to OCT examination. The patient's chin was positioned in the chin rest. The patient was asked to fixate on a target point inside the instrument the phase is completed by a camera, located inside the instrument that displays the fundus and scan beam. After the patient scanning was finished, analysis protocol was used to obtain circular maps on the macula.

\section{The steps of OCT imaging were done as follows:}

- Mydriatic eye drops Swixolate (Cyclopentolate Hydrochloride $10 \mathrm{mg} / \mathrm{ml}$ CHEMIPHARM) eye drops three times within 30 minutes were used to achieve as much pupil dilatation as we can to assure maximal OCT signal and analysis in patient's eyes prior to OCT examination.

- The patient's chin was positioned in the chin rest.

- The patient was asked to fixate on a target point inside the instrument the phase is completed by a camera, located inside the instrument that displays the fundus and scan beam.

- $\quad$ After the patient scanning was finished, analysis protocol was used to obtain circular maps on the fovea

(Figure 1).

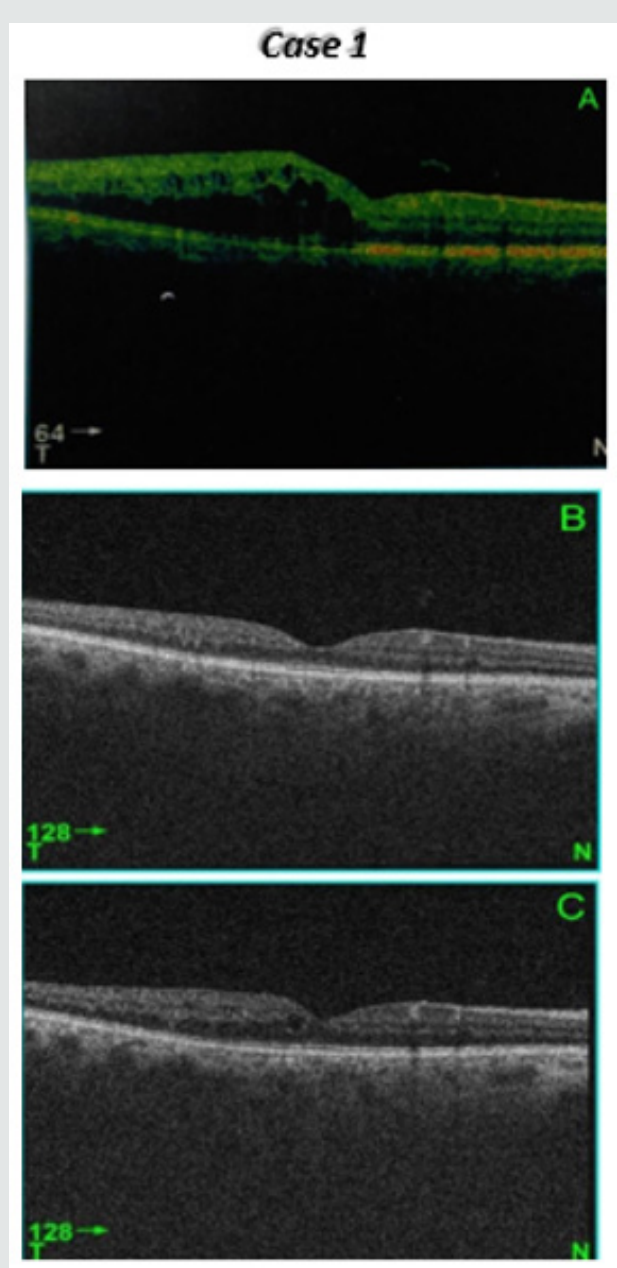

Figure 1: 46 years old male patient, hypertensive, phakic with right branch retinal vein occlusion showing diffuse macular edema with central macular thickness (CMT) 482 $\mu \mathrm{m}$.Gradual decrease of CMT at month 4 after the 3rd intravitreal ranizumab injection to $184 \mu \mathrm{m}$ and at month 6 to $208 \mu \mathrm{m}$. BCVA improved from base line logMAR 0.6 to 0.0 at month 4 and to 0.1 at month 6 after injection (follow up). 


\section{Interpretation}

Macular thickness was reported according to Early Treatment of Diabetic Retinopathy Study. Early Treatment Diabetic Retinopathy Study ring is a $6 \mu \mathrm{m}$ macular thickness map centered on the foveola that divided the macula into nine regions. It was divided into three rings, with the central ring corresponding to the fovea $(1 \mu \mathrm{m}$ diameter), the middle ring corresponding to the parafovea $(2 \mu \mathrm{m}$ diameter), and the outer ring corresponding to the perifovea ( $3 \mu \mathrm{m}$ diameter) and then divided into four quadrants, namely superior, nasal, inferior and temporal except for the central circle (Figure 2). Central macular thickness (CMT; foveal thickness) was defined as the average macular thickness in the central $1 \mu \mathrm{m}$, average macular thickness was defined as the mean of thicknesses in nine regions, and macular volume was defined as the sum of volumes in all nine regions. The macular retinal map divides the region into a central area with a radius of 500 microns, and two concentric rings inner parafoveal ring and outer perifoveal ring which were divided into four quadrants. The analysis program reports the corresponding mean thickness in each of the areas using assigned colors to indicate retinal thickness in the region under analysis. The analysis program reports the corresponding mean thickness in each of the areas using assigned colors to indicate retinal thickness in the region under analysis.

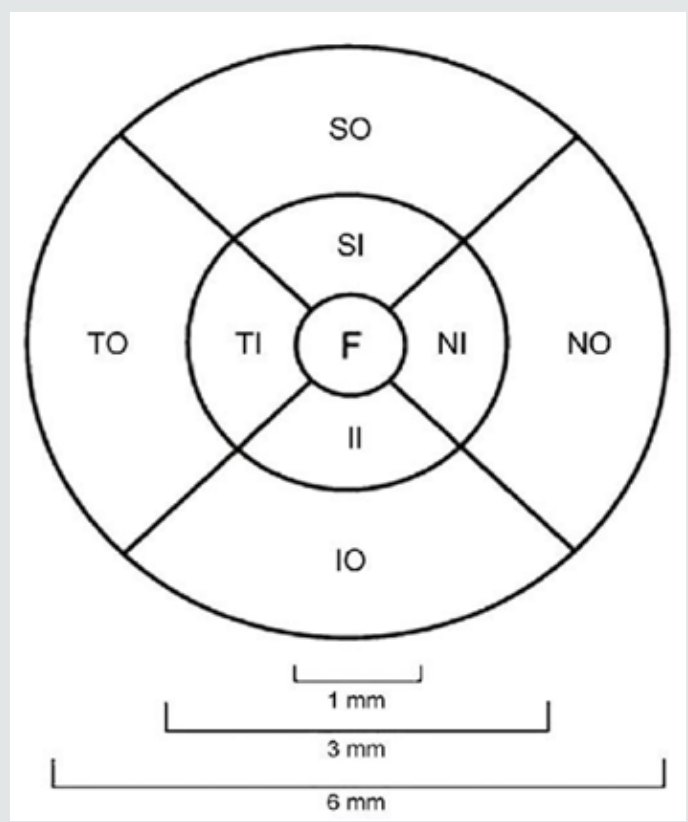

Figure 2: ETDRS ring and its divisions: $\mathrm{F}$ = fovea; $\mathrm{SI}=$ superior inner; $\mathrm{TI}=$ temporal inner; $\mathrm{II}=$ inferior inner; $\mathrm{NI}$ = nasal inner; $\mathrm{SO}=$ superior outer; $\mathrm{TO}=$ temporal outer; $\mathrm{IO}=$ inferior outer; $\mathrm{NO}=$ nasal outer (Kim et al., 2016).

\section{Intravitreal Injection of Ranibizumab}

\section{Treatment protocol}

Ranibizumab $(0.5 \mathrm{mg}, 0.05 \mathrm{~mL})$ was injected intravitreally under complete sterile conditions via the pars plana once monthly for 3 months.

\section{Treatment procedure}

Intravitreal injections were carried out, under aseptic conditions at mansoura ophthalmic center operating theater.

\section{Preoperative preparation}

Prophylactic topical antibiotic (Vigamox ED Q.I.D) on the day before the operative day. Pupil dilatation: One hour before surgery the pupil was dilated with cyclopentolate Hcl 1\% every 10 minutes for half an hour preoperatively.

\section{Preparation and Administration}

Ranibizumab (Lucentis) (Genentech/Roche, USA) is supplied as a preservative-free, colorless to pale yellow, sterile solution placed in a single-use glass vial. The dose is $0.5 \mathrm{mg}$ dose vial (delivers $0.05 \mathrm{~mL}$ of $10 \mathrm{mg} / \mathrm{mL}$ Ranibizumab). Lucentis should be inspected visually for particulate matter and discoloration prior to administration. The contents of a vial of ranibizumab should be drawn using a 19-gauge filter needle. A sterile small gauge $\mathrm{x} 1 / 2$ inch-needle should replace the filter needle for the injection.

\section{Follow Up}

Follow-up was one day and one week after injection and then every month for six months.

\section{Outcomes}

Outcomes included BCVA (functional response) and central foveal thickness (anatomical response),IOP and complications. Patient response were classified according to change in BCVA into good response in patients gaining more than 2lines on Snellen chart, moderate response in patients gaining less than 2lines and poor response in patients showing stable vision on chart with improvement of vision .

\section{Statistical analysis}

Data were analyzed with Statistical Package for the Social Sciences (SPSS) software package version 24.0 (Armonk, NY.IBM Cor).The normality of data was first tested with one-sample Kolmogorov-Smirnov test. Qualitative data were described using number and percent. Parametric data (normally distributed data) were described as mean (SD), non-parametric (non-normally distributed data) were described as median. Wilcoxon signed rank (for non-parametric data) was used to compare change within the same group pre and postoperative injection. P Level is considered statistically significant $<0.05$.

\section{Result}

Thirty-nine (19 CRVO and 20 BRVO) eyes of 39 patients were enrolled in the study with mean age of $56.56 \pm 9.6$ (range 24-69). $48.7 \%$ were male and $51.3 \%$ were females. Hypertension was detected in $69.2 \%$, Hyperlipidemia in $2.6 \%$. The mean best 
corrected visual acuity was $1.5 \log$ MAR, $1.00 \log$ MAR,1.00 log MAR, preoperative, fourth month, six months postoperative, respectively, ( $\mathrm{p}<0.001$ ) (Table 1) ( Figure 3). The mean central macular thickness was $675 \mu, 306 \mathrm{u}, 264 \mathrm{u}$, preoperative, fourth month, six months postoperative, respectively, ( $\mathrm{p}<0.001$ ) ( Table 2) ( Figure 4). The OP was $16.5 \mathrm{mmHg}, 16.8 \mathrm{mmHg}, 16.5 \mathrm{mmHg}$, preoperative, fourth month, six months postoperative, respectively, $(p=0.423)$. The mean BCVA in branch retinal vein occlusion is nearly the same in central retinal vein occlusion pre and postoperative while preoperative CMT is significantly higher in central retinal vein occlusion than branch retinal vein occlusion while postoperative there was significant improvement in CMT in both central and branch retinal vein occlusion but no statistically significant difference between both. The mean IOP in branch retinal vein occlusion is nearly the same in central retinal vein occlusion pre and postoperative with no statistically significant difference (Table 3).

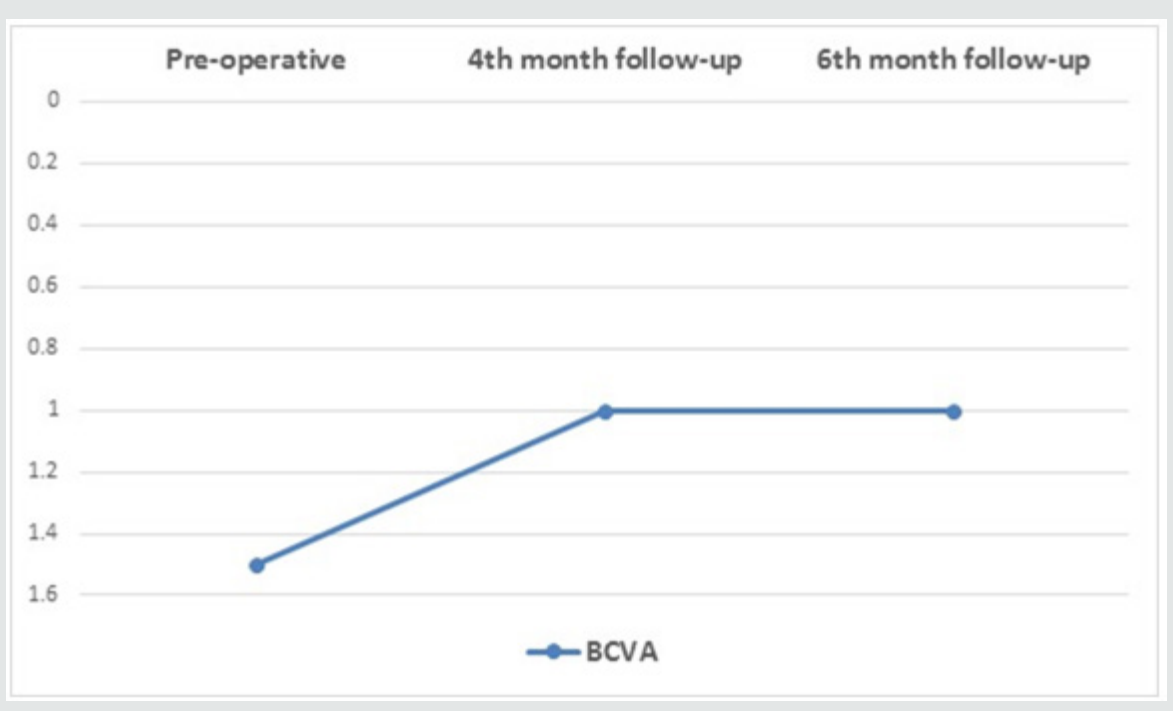

Figure 3: Changes in preoperative BCVA, $4^{\text {th }}$ month, $6^{\text {th }}$ month follow up.

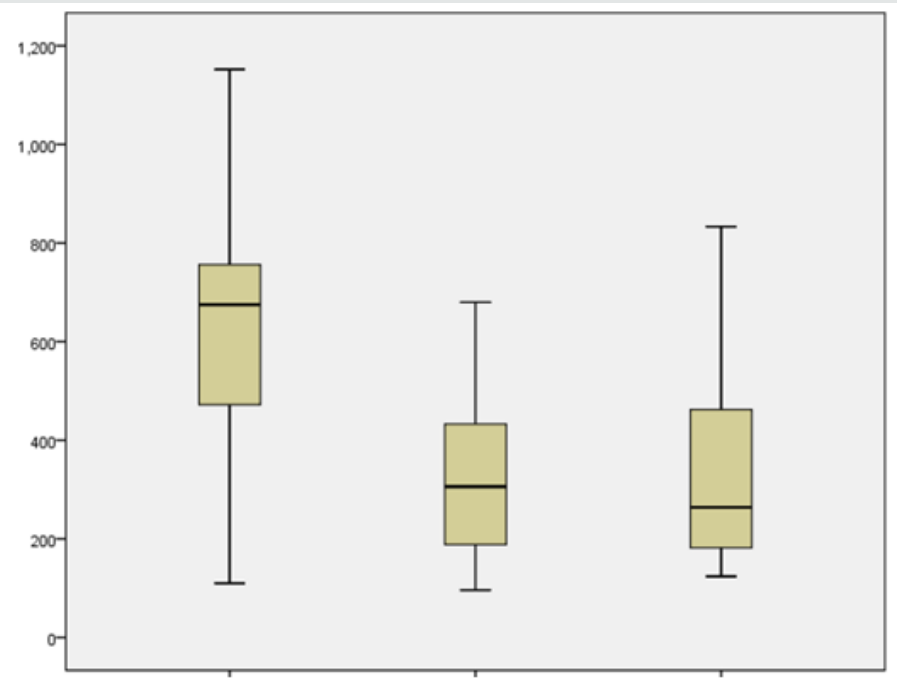

Figure 4: Comparison between preoperative CMT and $4^{\text {th }}$ month, $6^{\text {th }}$ month follow up.

Table 1: Comparison between baseline BCVA preoperative, $4^{\text {th }}$ month, $6^{\text {th }}$ month follow up.

\begin{tabular}{|c|c|c|c|c|c|c|}
\hline \multirow{2}{*}{ Items } & Pre-Operative & 4th month follow-up & 6th month follow-up & \multirow{2}{*}{ P1 } & \multirow{2}{*}{ P2 } & \multirow{2}{*}{ P3 } \\
\hline & Median (min-max) & Median (min-max) & Median (min-max) & & & \\
\hline BCVA & 1.5 & 1 & 1 & $\mathrm{Z1}=5.02$ & $\mathrm{Z} 2=5.01$ & $\mathrm{Z} 3=0.12$ \\
\hline “log MAR" & $(0.4-1.5)$ & $(0-1.5)$ & $(0-1.5)$ & $\mathrm{P} 1 \leq 0.001^{*}$ & $\mathrm{P} 2 \leq 0.001^{*}$ & $\mathrm{P} 3=0.9$ \\
\hline
\end{tabular}

Z1, P1: significance 0 pre-operative and 4th month follow-up (Z of Wilcoxon test). Z2, P2: significance () pre-operative and 6th month follow-up (Z of Wilcoxon test). Z3, P3: significance $04^{\text {th }}$ month and $6^{\text {th }}$ month follow-up (Z of Mann-Whitney U test). 
Table 2: Comparison between preoperative CMT, $4^{\text {th }}$ month, $6^{\text {th }}$ month follow up.

\begin{tabular}{|c|c|c|c|c|c|c|}
\hline \multirow{2}{*}{ Items } & Pre-Operative & $4^{\text {th }}$ month follow-up & $6^{\text {th }}$ month follow-up & \multirow{2}{*}{ P1 } & \multirow{2}{*}{ P2 } & \multirow{2}{*}{ P3 } \\
\hline & Median (min-max) & Median (min-max) & Median (min-max) & & & \\
\hline \multirow{2}{*}{ CMT } & 675 & 306 & 264 & $\mathrm{Z} 1=5.02$ & $\mathrm{Z} 2=4.54$ & $\mathrm{Z} 3=0.29$ \\
\hline & $(110-1152)$ & $(96-680)$ & (124-833) & $\mathrm{P} 1 \leq 0.001^{*}$ & $\mathrm{P} 1 \leq 0.001^{*}$ & P3 $=0.77$ \\
\hline
\end{tabular}

Z1, P1: significance () pre-operative and $4^{\text {th }}$ month follow-up (Z of Wilcoxon test). Z2, P2: significance () pre-operative and $6^{\text {th }}$ month follow-up (Z of Wilcoxon test). Z3, P3: significance $04^{\text {th }}$ month and $6^{\text {th }}$ month follow-up (Z of Mann-Whitney U test). CMT: central macular thickness

Table 3: Correlations between retinal vein occlusion (BRVO \& CRVO) and BCVA, CMT, IOP.

\begin{tabular}{|c|c|c|c|c|}
\hline Items & CRVO (n=19) & BRVO $(n=20)$ & Test of significance & $P$ Value \\
\hline $\begin{array}{l}\text { Pre-operative BCVA “log } \\
\text { MAR" }\end{array}$ & $1.5(0.4-1.5)$ & $1.6(0.4-1.5)$ & $\mathrm{Z}=0.55$ & 0.58 \\
\hline $4^{\text {th }}$ month BCVA “log MAR" & $1(0.3-1.5)$ & $1(0-1.5)$ & $\mathrm{Z}=0.01$ & 0.99 \\
\hline $6^{\text {th }}$ month BCVA “log MAR" & $1(0.3-1.5)$ & $1(0-1.5)$ & $Z=0.59$ & 0.55 \\
\hline Pre-operative CMT & $680.5(248-1152)$ & $523(110-969)$ & $\mathrm{Z}=1.89$ & $0.05^{*}$ \\
\hline $4^{\text {th }}$ month CMT & $344(127-680)$ & $305.5(96-614)$ & $\mathrm{Z}=0.96$ & 0.34 \\
\hline $6^{\text {th }}$ month CMT & $352.5(124-651)$ & $216(137-833)$ & $\mathrm{Z}=0.78$ & 0.44 \\
\hline Pre-operative IOP & 16.5 & 16.5 & $Z=0.67$ & 0.82 \\
\hline $4^{\text {th }}$ month IOP & 16.5 & $16.5(16.5-19.6)$ & $\mathrm{Z}=0.74$ & 0.69 \\
\hline $6^{\text {th }}$ month IOP & 16.5 & 16.5 & $\mathrm{Z}=0.67$ & 0.82 \\
\hline
\end{tabular}

Table 4: Spearman correlation between preoperative BCVA and other parameters.

\begin{tabular}{|c|c|c|}
\hline \multirow{2}{*}{ Items } & \multicolumn{2}{|c|}{ Pre-operative BCVA "log MAR" } \\
\cline { 2 - 3 } & $\mathbf{R}$ & 0.82 \\
\hline Age & 0.04 & $\leq 0.001^{*}$ \\
\hline VA pre-operative "log MAR" & 0.86 & $0.04^{*}$ \\
\hline CMT pre-operative & 0.33 & $\leq 0.001^{*}$ \\
\hline VA 1st month "log MAR" & 0.68 & $\leq 0.001^{*}$ \\
\hline BCVA 1st month "log MAR" & 0.6 & 0.21 \\
\hline CMT 1st month & 0.21 & $\leq 0.001^{*}$ \\
\hline VA 3rd month "log MAR" & 0.64 & $\leq 0.001^{*}$ \\
\hline BCVA 3rd month "log MAR" & 0.57 & 0.09 \\
\hline CMT 3rd month & 0.28 & 0.58 \\
\hline Duration of DM & 0.09 & 0.69 \\
\hline Duration of HTN & 0.07 & \multicolumn{2}{|c|}{} \\
\hline
\end{tabular}

Correlation coefficients and $\mathrm{P}$ values for each independent variable was calculated. Variables that had statistically significant positive correlations $(\mathrm{P}<0.05)$ with both log MAR BCVA and CMT were the following baseline BCVA and systemic risk factors had statistically significant negative correlations $(\mathrm{P}<0.05)$. Age, duration of diabetes mellitus, and duration of hypertension had statistically non-significant correlations with BCVA and CMT (Table 4) (Figure 5). Correlation coefficients and $P$ values for $6^{\text {th }}$ month postoperative BCVA and independent variables was calculated. Variables that had $6^{\text {th }}$ month best corrected visual acuity is significantly correlated with preoperative and $4^{\text {th }}$ month BCVA. Also significantly correlated to the $6^{\text {th }}$ month central macular thickness (Table 5). 


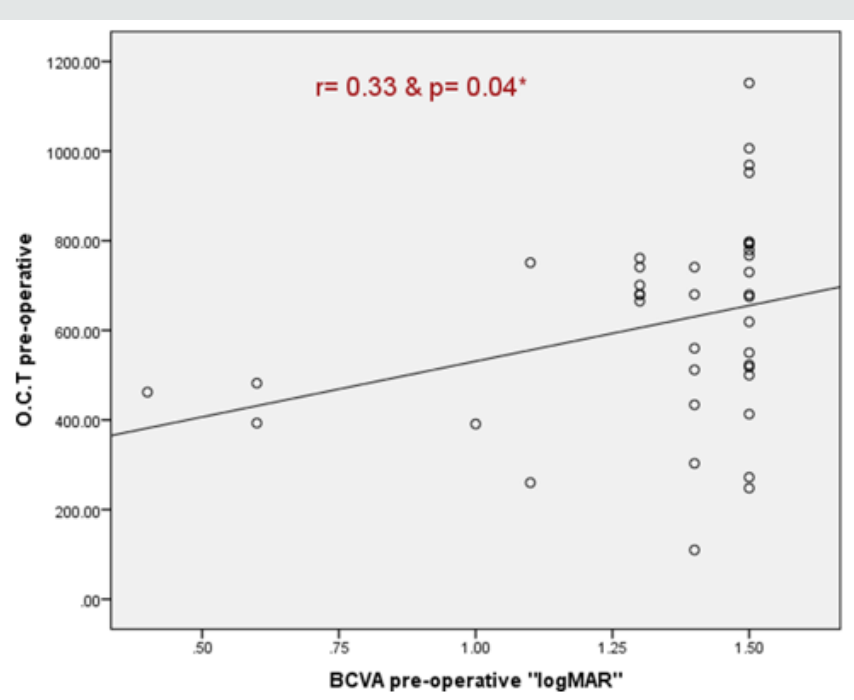

Figure 5: Correlation between pre-operative BCVA and preoperative CMT.

Table 5: Spearman correlation 6th month postoperative BCVA and other parameters.

\begin{tabular}{|c|c|c|}
\hline \multirow{2}{*}{ Items } & R & 6 $^{\text {th }}$ month postoperative BCVA “log MAR" \\
\cline { 2 - 3 } & 0.07 & 0.67 \\
\hline Age & 0.62 & $\leq 0.001^{*}$ \\
\hline VA pre-operative "log MAR" & 0.14 & 0.39 \\
\hline CMT pre-operative & 0.79 & $\leq 0.001^{*}$ \\
\hline BCVA 4 $4^{\text {th }}$ month “log MAR" & 0.24 & 0.15 \\
\hline CMT 4 $4^{\text {th }}$ month & 0.75 & $\leq 0.001^{*}$ \\
\hline CMT 6th month & 0.04 & 0.83 \\
\hline Duration of DM & 0.07 & 0.65 \\
\hline Duration of hypertension & $*$ significant $\mathrm{p} \leq 0.05$. r: correlation coefficient. \\
\hline
\end{tabular}

\section{Ocular side effects}

There were no observed significant ocular adverse events such as ocular inflammation, sterile and infectious endophthalmitis, vitreous hemorrhage, retinal detachment, or sustained increase in intraocular pressure with the use of intravitreal ranibizumab injections.

\section{Discussion}

Retinal vein occlusion (RVO) is a common vascular disorder of the retina and one of the most common causes of vision loss. It is the second most common cause of blindness from retinal vascular disease after diabetic retinopathy. RVO has been recognized as an entity since 1855, but many aspects of the pathogenesis and management of this disorder remain uncertain [8]. Macular edema is the most common cause of vision loss in patients with branch retinal vein occlusion or central retinal vein occlusion. Macular edema arises from breakdown of the BRB, resulting in the pathologic accumulation of both fluid and macromolecules in the retina. The breakdown of the BRB mediated by VEGF [9] and that intraocular levels of VEGF in the eyes with RVO are elevated [10].

The response to different anti-vascular endothelial growth factor treatments is not the same among patients despite they receive the same regimen [11]. Why some patients respond positively to treatment while the others do not is still a mystery. Studies have investigated and reported on factors such as age [12] smoking [13], and Genetic factors [14,15]. This was a prospective interventional study concentrated on short term visual and anatomical improvement after three loading doses of intravitreal Ranibizumab injections for macular edema secondary to retinal vein occlusion. Intravitreal Ranibizumab injections as monotherapy have shown short term promising results in both CRVO and BRVO, with a mean BCVA improvement as well as a decrease of central macular thickness [16]. Identifying predictive factors for good and poor visual outcomes with anti-VEGF therapy allows for more accurate prediction of prognosis based on the patient's baseline characteristics [17]. In the present study, after three loading doses, 
mean best corrected visual acuity improved from log MAR 1.5 to $1.00(\mathrm{p}<0.001)$. This is in agreement with studies [18-23]. There was significant reduction in central macular thickness from $675 \mu$ at baseline to $306 \mu$ at first month follow up ( $p<0.001)$ and to $264 \mu$ at third month follow up ( $p=0.77)$.This is an agreement with studies [18-23]. The present study found that ranibizumab is effective in improvement central macular thickness and best corrected visual acuity of the patients and the main improvement is statistically significant at 1 st month after injections.

The BRAVO and CRUISE trials are the first trials to show improvement in vision-related patient-reported outcomes in patients treated for macular edema following branch and central RVO, compared with no treatment. The reported reduced difficulty in performing vision-related activities was observed as early as 1 month after the first injection of ranibizumab in patients enrolled in both the BRAVO and CRUISE trials. Majority of the reported improvements in vision- related activities in ranibizumab treated patients were sustained in the months following the 6-month treatment period [21]. Pielen et al. [18] found that BCVA was reported from the baseline to follow-up at month 6 the results revealed that treatment with IVR $0.5 \mathrm{mg}$ significantly improved BCVA, compared with non-anti-VEGF $(\mathrm{P}<0.001)$ at six months. CMT is considered to be a strong prognostic measure for ME levels. At six months, CMT was significantly decreased $(\mathrm{P}<0.001)$ and this also agrees with the present study. Campochiaro et al. [10] found that at the primary endpoint in patients with macular edema due to RVO, the median change in visual acuity from baseline was 10 letters in 18 in the $0.5 \mathrm{mg}$ dose group. One patient in the $0.5 \mathrm{mg}$ dose group showed a reduction in VA of three letters at the primary endpoint, but all other patients showed improved vision. They also found that there was no correlation between the amount of improvement in VA and patient age as found in the current study. Wolf- Schnurrbusch et al. [20] revealed that the response to ranibizumab at four weeks after the first anti-VEGF injection, mean BCVA ranged from 20 to 86 letters (mean, $53 \pm 17$ letters). These results tunes with our results which explain that the main response of ranibizumab at one month after injection. Puche et al. [19] made the patients received a mean of 2.3 injections (range, 1-4 injections). Mean follow-up was 7 months (range, 2-20 months).They found that the marked functional and anatomical improvement was after the first injection with slight improvement after the rest of injections. They also found that the factors associated with final visual acuity of 20/40 or best were better baseline visual acuity $(\mathrm{p}<0.001)$ and thinner final central retinal thickness (CRT) $(p=0.009)$ but not baseline CRT $(p=0.440)$ nor the number of injections $(\mathrm{p}=0.153)$.

Pielen et al. [18] also found that CMT at month 3 was reduced by $379.5 \mu$ (204.2-554.8) and during the observation period from month 3 to month 6 mean CMT showed increase about $37.16 \mu$.The 3 months result is in coordnance with our study and It shows almost the same results during the next 3 months. They also found that the difference in BCVA is small (+17.5 letters/3 months and +17 letters/6 months versus 18.3 letters). Spaide et al. [24] reported that VA showed a mean improvement of 10.4 letters over baseline $(\mathrm{P}=.001)$, and there was a decrease of the CMT to $199 \mu \mathrm{m}(\mathrm{P}<.001)$ and this was slightly less than our study. At 12 months of followup, the mean VA improved to 64.3 letters (median, 68.5 letters), and the CMT decreased to $186 \mu \mathrm{m}(\mathrm{P}<.001)$. The change in CMT at one week was correlated with the change in macular thickness at 12 months $(\mathrm{P}<.001)$. The change in CMT at one month showed a smaller correlation with the change in CMT at 12 months $(\mathrm{P}=$ .047). Of interest is that the VA at one month was not predictive of the one-year outcome, so a single injection followed by a waitand-see approach is likely to be a suboptimal strategy. It may be possible that the best strategy is a repeated periodic injection given at intervals found to prevent any recrudescence of retinal pathologic features [24]. Kinge B et al. (23) evaluated 32 patients treated with monthly injections of intravitreal ranibizumab for three consecutive months and after that if there was persistant macular edema patients received further injections. In these patients the change of CMT from baseline was $292 \mu( \pm 226 \mu)$, p (0.001) at one month and $86 \mu( \pm 165 \mu), p(.071)$ at three months and that agrees with our study. The present study study found that central macular thickness had statistically significant positive correlations $(\mathrm{P}<0.01)$ with best corrected visual acuity. The results of current study are close to the results reported by long term studies that had previously investigated the outcome in patients with macular edema secondary to retinal vein occlusion undergoing ranibizumab therapy. This is may be explained by that ranibizumab main response is in the first month after injection and for better results and to decrease recurrence patients may need more than 3 injections [22].

In addition, both functional and anatomical improvement and the results have gone nearly hand in hand with the studies showing functional and anatomical improvement after intravitreal ranibizumab for 6 months and 12 months in many studies as [2124]. In the present study presence of systemic risk factor or the age had no statistically significant correlation with patient response to the treatment .That was also found in many studies as in [18, 22]. In the current study no, significant ocular adverse effects occurred such as endophthalmitis, cataract, retinal detachment and increase IOP, or systemic arterial thromboembolic events. This is consistent with Spaide et al. 2009 [24]. The BRAVO study assessed the safety and efficacy profile of Lucentis® in a total of 397 patients with macular edema following BRVO showed sustained vision improvement during the six-month study, with an effect seen as early as seven days and a benefit nearly maintained throughout the 12 months of follow-up [16]. BRIGHTER and CRYSTAL studies reported that a rapid and statistically significant decrease from baseline in central macular thickness was observed at one month 
and maintained up to month 24. The effect of ranibizumab treatment was similar irrespective of the presence of retinal ischemia. In BRIGHTER, patients with ischemia present $(\mathrm{N}=46)$ or absent $(\mathrm{N}=133)$ and treated with ranibizumab monotherapy, at Month 24. In CRYSTAL, patients with ischemia present $(\mathrm{N}=53)$ or absent $(\mathrm{N}=300)$ and treated with ranibizumab monotherapy . The long-term safety profile of ranibizumab observed in the 24-month studies is consistent with the known Lucentis safety profile $[25,26]$.

\section{Declarations}

Ethics approval and consent to participate; This interventional study was approved by the ethics committee of Faculty of medicine, Mansoura university (Registration Number: MS/16.02.108), Date:) and adhered to the tents of Declaration of Helsinki. All subjects provided written informed consent prior to study participation.

\section{Consent for Publication: Not applicable}

Availability of Data and Materials: Data are available upon request

Competing of interests: The authors declare that they have no conflict of interest

Funding: Authors have not declared a specific grant from this research from any funding agency in the public, commercial.

Authors contribution: GA and EM designed the study, EM and ES performed examinations of individuals, HS prepared and carried out the analysis, ES and GW interpreted and discussed the results, and wrote the first version of the manuscript. All authors read and approved the final manuscript.

\section{Acknowledgement: Not applicable}

\section{References}

1. A Jaulim, B Ahmed, T Khanam IP Chatziralli (2013) Branch retinal vein occlusion: epidemiology, pathogenesis, risk factors, clinical features, diagnosis and complications. Retina 33(5): 901-910.

2. Niral K (2010) Retinal vein occlusion: pathophysiology and treatment options. Clin Ophthalmol 4: 809-816.

3. Noma H, Minamoto A, Funatsu H, Tsukamoto H, Nakano K, et al. (2006) Intravitreal levels of vascular endothelial growth factor and interleukin-6 are correlated with macular edema in branch retinal vein occlusion. Graefes Arch Clin Exp Ophthamol 244(3): 309-315.

4. Kimberly S, Samantha F, Thomas H, AC Andrew (2019) Five years outcome of retinal vein occlusion treated with vascular endothelial growth factor inhibitors. BMJ open Ophthalmol 4(1): e000249.

5. Alex Y and Rishi S (2011) Ranibizumab for the treatment of macular edema following retinal vein occlusion. Clin Investig 1(9).

6. Shorya VA, Amjad S, Deepankur M, Siddharth S Rajvardhan A (2014) Comparative evaluation between Ranibizumab combined with Laser and Bevacizumab combined with Laser versus Laser alone for macular oedema secondary to branch retinal vein occlusion. MEA J Ophthalmol 21(4): 296-301.

7. Tasanee Braithwaite, Afshan A Nanji, Kristina Lindsley, Paul B Greenberg (2014) Anti-vascular endothelial growth factor for macular oedema sec- ondary to central retinal vein occlusion. Cochrane Database Syst Rev 5(5): CD007325.

8. Jia Li, Yannis M Paulus YS, Wangyi F, Qinghuai L, Songtao Y (2017) New developments in the classification, pathogenesis, risk factors, natural history, and treatment of branch retinal vein occlusion. J Ophthalmol 2017: 4936924

9. William R, Drew D, Quan DN, Diana V Do (2017) Management of macular edema due to central retinal vein occlusion-The role of aflibercept. Taiwan J Ophthalmol 7(2): 70-76.

10. Campochiaro PA, Hafiz G, Shah SM, Nguyen QD, Ying H, et al. (2009) Ranibizumab for macular edema due to retinal vein occlusions: implication of VEGF as a critical stimulator. Mol Ther 16(4): 791-799.

11. Stefan C, Iliescu DA, Timaru CM, Schmitzer S, De Simone A, et al. (2015) Anti-vascular endothelial growth factor indications in ocular disease. Rom J Ophthalmol 59(4): 235-242.

12. Boyer DS, Antoszyk AN, Awh CC, Bhisitkul RB, Shapiro H, et al. (2007) Subgroup analysis of the MARINA study of ranibizumab in neovascular age-related macular degeneration. Ophthalmology 114(2) :246-252.

13. Kaiser PK, Brown DM, Zhang K, Hudson HL, Holz FG, et al. (2007) Ranibizumab for predominantly classic neovascular age-related macular degeneration: subgroup analysis of first-year ANCHOR results. Am J Ophthalmol 144(6): 850-857.

14. Teper SJ, Nowinska A, Pilat J, Palucha A Wylegala E (2010) Involvement of genetic factors in the response to a variable-dosing ranibizumab treatment regimen for age-related macular degeneration. Mol Vis 16: 2598-2604.

15. Tian J, Qin X, Fang K, Chen Q Hou J, et al. (2012) Association of genetic polymorphisms with response to bevacizumab for neovascular age-related macular degeneration in the Chinese population. Pharmacogenomics 13(7): 779-787.

16. Pereira H, Henriques J, Fonseca P, Cachulo ML, Pires I, et al. (2011) Intravitreal Ranibizumab (Lucentis ${ }^{\circledR}$ ) for the Treatment of Macular Edema of Central and Branch Retinal Vein Occlusion. Oftalmologia 35: 327-334.

17. Singh RP, Fu EX, Smith SD, Williams DR, Kaiser PK (2009) Predictive factors of visual and anatomical outcome after intravitreal bevacizumab treatment of neovascular age-related macular degeneration: an optical coherence tomography study. Br J Ophthalmol 93(10): 1335-1353.

18. Pielen A, Mirshahi A, Feltgen N, Lorenz K, Korb C, et al. (2015). Ranibizumab for Branch Retinal Vein Occlusion Associated Macular Edema Study (RABAMES): six-month results of a prospective randomized clinical trial. Acta ophthalmologica 93(1): e29-e37.

19. Puche N, Glacet A, Mimoun G, Zourdani A, Coscas G (2012) Intravitreal Ranibizumab for macular oedema secondary to retinal vein occlusion: a retrospective study of 34 eyes. Acta ophthalmologica 90(4): 357-361.

20. Wolf EJ, Braunstein A, Shih C, Braunstein RE (2007) Incidence of visually significant pseudo phakic macular edema after uneventful phacoemulsification in patients treated with nepafenac. J Cat Ref Surg 33(9): 15461549.

21. Varma R, Bressler NM, Suñer I, Lee P, Dolan CM, et al. (2012) Improved vision-related function after ranibizumab for macular edema after retinal vein occlusion: results from the BRAVO and CRUISE trials. Ophthalmology 119(10): 2108-2118.

22. Campochiaro PA, Heier JS, Feiner L, Gray S, Saroj N, et al. (2010) Ranibizumab for macular edema following branch retinal vein occlusion: six-month primary end point results of a phase III study. Ophthalmology 117(6): 1102-1112.

23. Kinge B, Stordahl PB, Forsaa V, Fossen K, Haugstad M, et al. (2010) Efficacy of ranibizumab in patients with macular edema secondary to central retinal vein occlusion: results from the sham controlled ROCC study. Am J Ophthalmol 150(3): 310-314. 
24. Spaide RF, Chang LK, Klancnik JM, Yannuzzi LA, Sorenson J, et al. (2009) Prospective study of intravitreal ranibizumab as a treatment for decreased visual acuity secondary to central retinal vein occlusion. Am J Ophthalmol 147(2): 298-306.

25. Ramin T, Sebastian MW, Francesco B, Heinrich G, Margarita G, et al. (2017) Sustained benefits of Ranibizumab with or without Laser in branch retinal vein occlusion: 24-month results of the BRIGHTER study. Ophthalmology 124(12): 1778-1787.

26. Michael L, Sebastian W, Siegfried P ,Philip H, Elizabeth B, et al. (2018) Sustained benefits from Ranibizumab for central retinal vein occlusion with macular edema: 24-month results of the CRYSTAL study. Ophthalmology 125(3): 134-142.

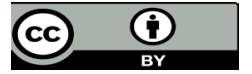

This work is licensed under Creative Commons Attribution 4.0 License

To Submit Your Article Click Here:

Submit Article

DOI: $10.32474 /$ TOOAJ.2020.03.000153 